\title{
Postoperative prophylactic hepatic arterial infusion chemotherapy for stage III colorectal cancer: a retrospective study
}

This article was published in the following Dove Press journal:

OncoTargets and Therapy

26 September 2016

Number of times this article has been viewed

\author{
Yao Wang' \\ Xin Rong Sun' \\ Wen Ming Feng' \\ Ying Bao' \\ Yin Yuan Zheng² \\ 'Department of General Surgery, \\ ${ }^{2}$ Department of Radiology, First \\ People's Hospital affiliated to Huzhou \\ University Medical College, Huzhou, \\ People's Republic of China
}

Correspondence: Yin Yuan Zheng

Department of Radiology, First People's Hospital affiliated to Huzhou University Medical College, 158 Guangchang Hou Road, Huzhou, Zhejiang 313000, People's Republic of China

Tel +865722039409

Fax +86 5722023728

Email yinyuan_zheng@sina.com
Background: Radical resection is the main treatment for colorectal cancer (CRC), but metastasis or recurrence is common in which liver metastasis accounted for $83 \%$ of the cases. Therefore, the prognosis of patients with advanced CRC may be improved if liver metastasis is prevented. This study aims to investigate the efficacy of hepatic arterial infusion chemotherapy (HAIC) on liver metastases of stage III CRC patients after curative resection.

Methods: Between 2002 and 2008, 287 stage III CRC patients who had undergone radical resection were included in this study. According to postoperative adjuvant chemotherapy modality, these patients were divided into two groups. Patients in the combined therapy group received two cycles of HAIC plus four cycles of systemic chemotherapy, while patients in the monotherapy group received six cycles of systemic chemotherapy alone. The HAIC regimen consisted of hepatic arterial infusion of oxaliplatin (OXA, $85 \mathrm{mg} / \mathrm{m}^{2}$ ) on day 1 and 5-fluorouracil (5-FU, $2,400 \mathrm{mg} / \mathrm{m}^{2}$ ) on days 2 and 3 followed by a vein infusion of folinic acid (FA, $200 \mathrm{mg} / \mathrm{m}^{2}$ ) as a 2-hour infusion on days 2 and 3. The systemic chemotherapy regimen consisted of a 2-hour infusion of OXA $\left(85 \mathrm{mg} / \mathrm{m}^{2}\right)$ on day 1 followed by FA $\left(200 \mathrm{mg} / \mathrm{m}^{2}\right)$ as a 2-hour infusion on days 2 and 3, and by 5 -FU $\left(2,400 \mathrm{mg} / \mathrm{m}^{2}\right)$ as a 48 -hour infusion. This was repeated every 4 weeks. All cases were followed up for 5 years or until death. The 5 -year overall survival, disease-free survival, liver metastases-free survival, and the overall liver metastases rates were retrospectively compared.

Results: Significant differences were found in the 5-year overall survival (combined therapy, $70.71 \%$; monotherapy, $57.14 \%$; $P=0.014$ ), disease-free survival (combined therapy, $69.29 \%$; monotherapy, $55.78 \% ; P=0.021$ ), and liver metastases-free survival rates (combined therapy, $70 \%$; monotherapy, $56.46 \% ; P=0.019)$.

Conclusion: Prophylactic adjuvant HAIC can prevent metachronous liver metastases and improve the prognosis of patients with stage III CRC after curative resection.

Keywords: colorectal cancer, hepatic arterial infusion chemotherapy, liver metastases, chemotherapy

\section{Introduction}

Colorectal cancer ( $\mathrm{CRC}$ ) has become a major cause of morbidity and mortality worldwide. ${ }^{1}$ Currently, the newly diagnosed cases in the world are over one million per year. ${ }^{2}$ Radical resection is the main treatment for CRC, but metastasis or recurrence is common in which liver metastasis accounted for $83 \%$ of the cases. ${ }^{3}$ Therefore, prevention of liver metastases can be expected to improve most effectively the prognosis of patients with advanced CRC. Multiple trials over the past decades have shown that hepatic arterial infusion chemotherapy (HAIC) is an effective treatment 
for liver metastases from CRC with superior response rates (RRs) compared with systemic or portal vein infusion chemotherapy. ${ }^{4}$ Moreover, a pilot study proved that preventive adjuvant HAIC with 5-fluorouracil (5-FU) could effectively prevent metachronous liver metastasis from CRC after curative resection compared with nontreated patients. ${ }^{5}$ However, it is still unclear whether prophylactic adjuvant HAIC is superior to systemic chemotherapy in the prevention of liver metastasis.

Oxaliplatin (OXA) is a new platinum compound with significant activity in CRC. For patients with CRC, adding OXA to 5-FU/folinic acid (FA) has produced greater RRs and overall survival (OS) ${ }^{6,7}$ In a rabbit tumor model, intraarterial (ia) hepatic OXA has shown a significant pharmacokinetic advantage compared with intravenous (iv) infusion. ${ }^{8}$ Several Phase I and II studies of HAI with OXA have been published to date. Tolerability and efficacy in heavily pretreated patients have been demonstrated; in particular, a liver extraction of OXA above 50\% with minimal systemic concentration and toxicity has been reported. ${ }^{9,10}$ The aim of this study was to determine the efficacy of OXA/FA/5-FU administrated by hepatic arterial infusion (HAI) in patients with stage III CRC after curative resection.

\section{Methodology}

\section{Patients}

A total of 287 cases of stage III CRC patients who underwent radical resection in the First People's Hospital affiliated to Huzhou University Medical College between 2002 and 2008 were enrolled in this retrospective study. The inclusion criteria were as follows: verified carcinoma of the colon or the rectum, stage III (any T, N1 or N2, M0) disease by operation and pathology (according to the 1997 revision of the International Union Against Cancer tumor-node-metastasis [TNM] staging system); no prior cancer therapy; no portal vein thrombosis; performance status tolerated HAIC and systemic chemotherapy with Eastern Cooperative Oncology Group 0-2; modest hepatic and renal function; adequate bone marrow, and age 18-75 years. ${ }^{11}$ Ultrasonography or computed tomography (CT) was also performed to confirm that there were no liver metastases within 6 months after operation. The exclusion criteria were as follows: anal cancer; received neoadjuvant chemotherapy or radiotherapy; recurrence, metastasis, or death within 6 months after operation; a history of other malignancies.

According to postoperative adjuvant chemotherapy modality, these patients were assigned to two groups: combined therapy group (patients received two cycles of HAIC plus four cycles of systemic chemotherapy) and monotherapy group (patients received six cycles of systemic chemotherapy alone).

This study was conducted in accordance with the principles of the Declaration of Helsinki and "Good Clinical Practice" guidelines. A written informed consent was obtained from all patients. Approval of the study was obtained from the institutional research ethics committee of First People's Hospital affiliated to Huzhou University Medical College.

\section{Chemotherapy administration}

Patients received two cycles of HAIC followed by four cycles of systemic chemotherapy (combined therapy) or six cycles of systemic chemotherapy alone (monotherapy). The HAIC regimen consisted of HAI of OXA $\left(85 \mathrm{mg} / \mathrm{m}^{2}\right)$ on day 1 and 5 -FU $\left(2,400 \mathrm{mg} / \mathrm{m}^{2}\right)$ on days 2 and 3 followed by a vein infusion of FA $\left(200 \mathrm{mg} / \mathrm{m}^{2}\right)$ as a 2 -hour infusion on days 2 and 3 . After local anesthetic administration, a catheter was inserted through the right femoral artery using the Seldinger method. Arteriography of the celiac trunk was performed to reveal the hepatic arterial anatomy. The tip of the catheter was placed at the common hepatic artery or proper hepatic artery. The other end of the catheter was properly fixed and connected to the injection port of a micropump. The catheter was removed after infusion. The systemic chemotherapy regimen consisted of a 2-hour infusion of OXA $\left(85 \mathrm{mg} / \mathrm{m}^{2}\right)$ on day 1 followed by FA $\left(200 \mathrm{mg} / \mathrm{m}^{2}\right)$ as a 2 -hour infusion on days 2 and 3 , and by 5 -FU $\left(2,400 \mathrm{mg} / \mathrm{m}^{2}\right)$ as a 48 -hour infusion.

This study prophylactically used 5-HT3-receptor antagonist granisetron ( $8 \mathrm{mg}$ ) iv for antiemetic treatment, and administered dexamethasone ( $5 \mathrm{mg}$ ) iv prior to the application of OXA and also during 5-FU infusion. The patients received treatment every 4 weeks. Treatment was continued for six cycles in the case of clinical response or until the disease progression, unacceptable toxicity/complication, or in accordance with patient's choice.

\section{Assessment and follow-up}

Complication and toxicity were analyzed according to medical records. Patients were followed up until the last follow-up or death. They were assessed every 2 weeks during treatment, every month during the first postoperative year, and then every 3 months thereafter. The baseline assessment also involved a medical history taking, physical examination, biological tests, measurement of the carcinoembryonic antigen (CEA) level, chest CT, and abdominal ultrasonography or CT. If recurrence or metastasis was found, they would be followed up every month and be treated in the following time periods. The diagnosis of recurrence or metastasis was 
made on the basis of imaging and, if necessary, cytological analysis or biopsy. A rise in CEA level alone was not accepted as evidence of relapse. The patients who were diagnosed with recurrence or metastasis would receive chemotherapy, radiofrequency ablation, or undergo surgery.

\section{Statistical analysis}

All measurements were expressed as mean \pm standard deviation. Statistical analyses were performed using the twosample $t$-test and adjusted chi-square test for the two groups. The exact chi-square test was also used if the individual cell size was less than five counts. Ridit analysis was used to compare the distributions of ranked data. Long-term survival was analyzed using the Kaplan-Meier method, and survival curves were compared by the log-rank test. $P$-value $<0.05$ was considered to be statistically significant.

\section{Results}

\section{Patient characteristics}

Patient characteristics are shown in Table 1. There was no significant difference between the two groups in terms of sex, age, location of tumor, T-stage of tumor, N-stage of tumor, tumor pathology, tumor size, and serum CEA level.

Table I Patient characteristics

\begin{tabular}{|c|c|c|c|}
\hline Characteristics & $\begin{array}{l}\text { Combined } \\
\text { therapy }(n=\mid 40)\end{array}$ & $\begin{array}{l}\text { Monotherapy } \\
(n=\mid 47)\end{array}$ & $P$-value \\
\hline Sex & & & 0.5668 \\
\hline Male & 83 & 92 & \\
\hline Female & 57 & 55 & \\
\hline Location & & & 0.6685 \\
\hline Colon & 89 & 97 & \\
\hline Rectum & 51 & 50 & \\
\hline T-stage of cancer & & & 0.2302 \\
\hline TI & 26 & 32 & \\
\hline $\mathrm{T} 2$ & 28 & 36 & \\
\hline $\mathrm{T} 3$ & 59 & 55 & \\
\hline $\mathrm{T} 4$ & 27 & 24 & \\
\hline $\mathrm{N}$-stage of cancer & & & 0.2925 \\
\hline $\mathrm{NI}$ & 82 & 95 & \\
\hline N2 & 58 & 52 & \\
\hline Pathology & & & 0.8821 \\
\hline Well & 21 & 25 & \\
\hline Moderately & 92 & 90 & \\
\hline Poorly & 21 & 22 & \\
\hline Mucinous & 6 & 10 & \\
\hline Age (years) & $64.3 \pm 14.2$ & $65.2 \pm 16.6$ & 0.6228 \\
\hline Tumor size (mm) & $48.3 \pm 26.2$ & $54.2 \pm 28.9$ & 0.0715 \\
\hline Serum CEA level (ng/mL) & $15.7 \pm 12.9$ & $18.6 \pm 13.2$ & 0.0610 \\
\hline Lost to follow-up & 0 & 0 & - \\
\hline
\end{tabular}

Note: Data presented as $\mathrm{n}$ or mean \pm standard deviation. Abbreviation: CEA, carcinoembryonic antigen.

\section{Five-year disease-free survival}

At the time of analysis, 65 patients in the monotherapy group had relapsed or died, as compared with 53 patients in the combined therapy group. The hazard ratio for recurrence in the monotherapy group, as compared with the combined therapy group, was 1.61 (95\% confidence interval, 1.06-2.52). The probability of disease-free survival at 5 years was $69.29 \%$ in the combined therapy group and $55.78 \%$ in the monotherapy group ( $P=0.021$ by log-rank test) (Figure 1$)$.

\section{Five-year overall survival}

At the time of the cutoff date of analysis, 63 patients had died in the monotherapy group, as compared with 41 patients in the combined therapy group. The hazard ratio for recurrence in the monotherapy group, as compared with the combined therapy group, was 1.59 (95\% confidence interval, 1.04-2.48). The probability of survival at 5 years was $70.71 \%$ in the combined therapy group and $57.14 \%$ in the monotherapy group ( $P=0.014$ by log-rank test) (Figure 2 ).

\section{Five-year liver metastases-free survival}

During the first 3 years after surgery, 28 of 140 patients in the combined therapy group and 45 of 147 patients in the monotherapy group developed liver metastases. The 5-year actuarial rate of liver metastases-free survival was $70 \%$ in the combined therapy group and $56.46 \%$ in the monotherapy group ( $P=0.019$ by log-rank test) (Figure 3 ).

\section{Complication and toxicity}

No treatment-related death occurred. All patients completed six cycles of monotherapy or combined therapy. All complications or toxicities were controlled by conservative treatment. Inguinal hematoma was observed in two patients from the combined therapy group and controlled by pressure bandage. No life-threatening toxicity or complication was observed. None of the patients required dose reduction.

\section{Discussion}

Liver is commonly the first site of distant metastasis and the major target organ of metastasis from CRC. ${ }^{12}$ Liver metastasis is the leading cause of death in CRC. ${ }^{13}$ Despite undergoing radical resection of the cancer, over half of the patients with stage III CRC develop liver metastases. ${ }^{14}$ Malignancy with liver metastasis plays an important role in daily oncology practice, ${ }^{15}$ especially in CRC. The authors' believe that a reduction in the incidence of liver metastases may be the most effective way of reducing the likelihood of recurrence and for improving the prognosis. 


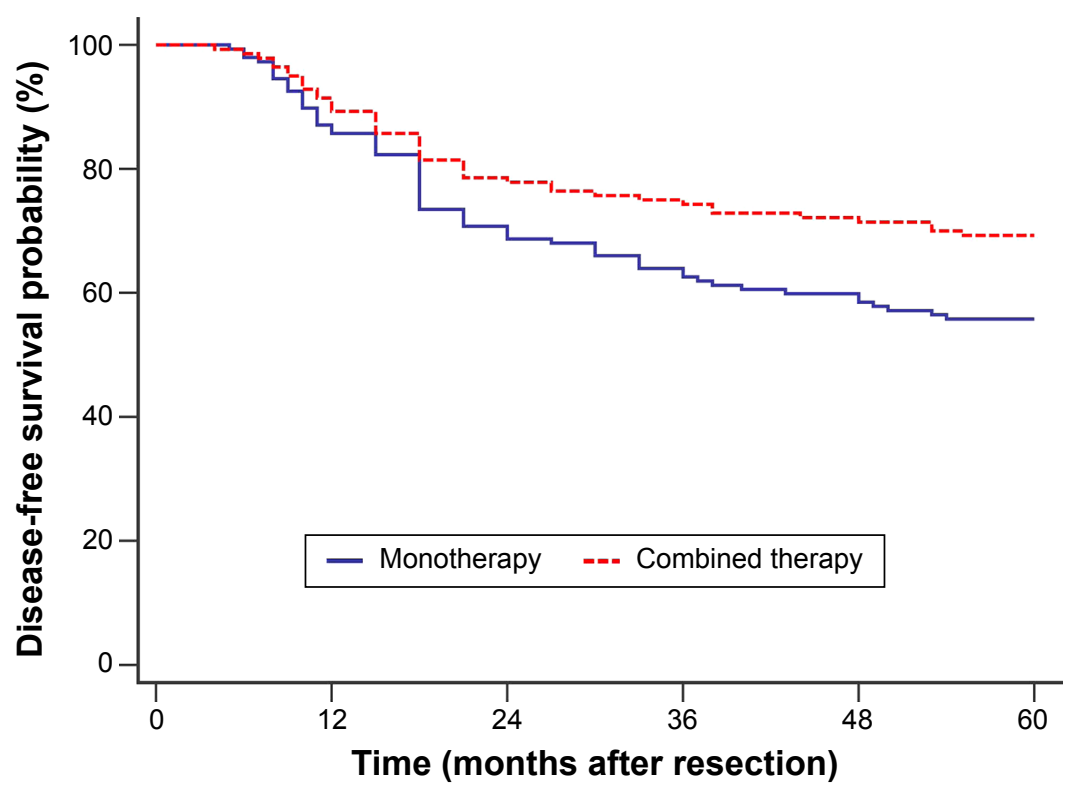

Figure I Disease-free survival curves after curative resection of stage III colorectal cancer. The probability of disease-free survival at 5 years was $69.29 \%$ in combined therapy group and $55.78 \%$ in the monotherapy group $(P=0.021$ by log-rank test).

In patients without liver metastasis at the time of surgery and judged by the surgeon to have been curatively resected, the metachronous liver metastasis occurring postoperatively is believed to originate from micrometastases present at the time of surgery. ${ }^{16}$ Previously, portal vein injection of 5-FU was used at the time of surgery and during the postoperative period to prevent metachronous liver metastasis. ${ }^{17}$ However, a large randomized trial showed that portal vein injection of 5 -FU had no significant impact on survival. ${ }^{18}$

With regard to liver metastases from $\mathrm{CRC}$, a meta-analysis of seven randomized controlled trials has suggested that
HAIC of 5-FU has a significantly better RR than systemic chemotherapy. ${ }^{4}$ It has been shown experimentally that liver tumors receive abundant blood supply from the hepatic artery. ${ }^{19-21}$ Therefore, HAIC seems to be an attractive therapeutic modality because extraction of cytotoxic drugs from the hepatic arterial circulation via the first-pass effect can result in high local concentrations and minimal systemic toxicity. ${ }^{22}$ In this study, patients in the combined therapy group were found to have a significantly better disease-free survival, overall survival, and liver metastases-free survival than those in the monotherapy group.

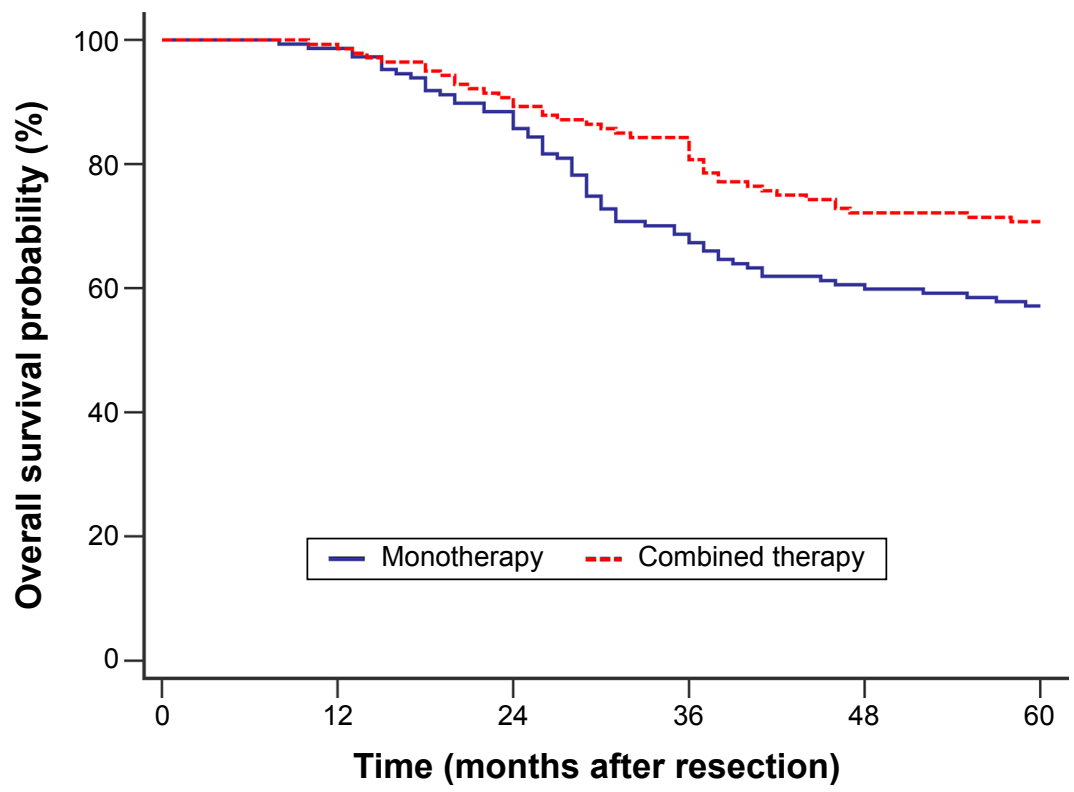

Figure 2 Overall survival curves after curative resection of stage III colorectal cancer. The probability of overall survival at 5 years was 70.7 I\% in the combined therapy group and $57.14 \%$ in the monotherapy group $(P=0.014$ by log-rank test). 


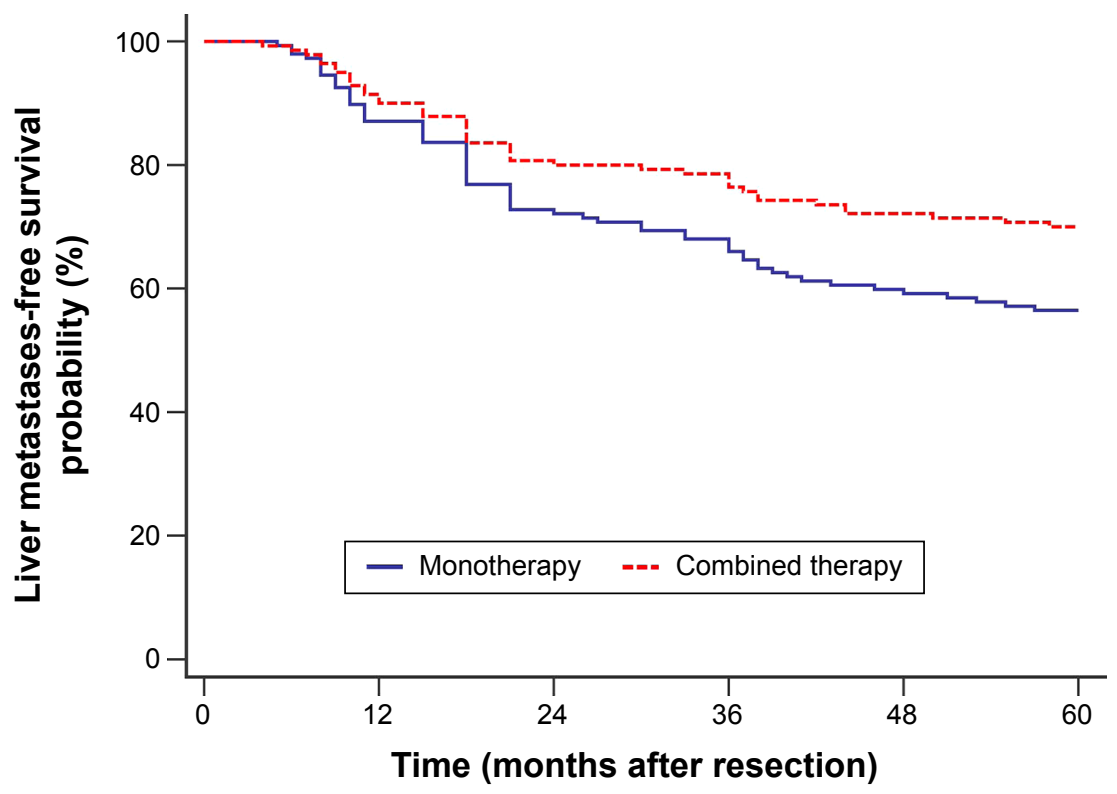

Figure 3 Liver metastases-free survival curves after curative resection of stage III colorectal cancer. The 5 -year actuarial rate of liver metastases-free survival was $70.00 \%$ in the combined therapy group and $56.46 \%$ in the monotherapy group ( $P=0.019$ by log-rank test).

The reason may be that HAIC eliminates the micrometastases which would later develop to metachronous liver metastasis after surgery. Moreover, no treatment-related death, life-threatening toxicity, or complication was observed in this study.

\section{Conclusion}

Prophylactic adjuvant HAIC can effectively and safely prevent metachronous liver metastases and improve the prognosis of patients with stage III CRC after curative resection. However, because of the retrospective nature and the small sample size, further prospective study with large sample size is needed to confirm the results of this study.

\section{Acknowledgment}

This study was supported by Foundation of Huzhou Bureau of Science and Technology (number 2011YS26).

\section{Disclosure}

The authors report no conflicts of interest in this work.

\section{References}

1. Yao Y, Zhao H, Sun Y, Lin F, Tang L, Chen P. Combined chemotherapy of hydroxycampothecin with oxaliplatin as an adjuvant treatment for human colorectal cancer. Tohoku J Exp Med. 2008;215(3): 267-278.

2. Nordlinger B, Sorbye H, Glimelius B, et al. Perioperative chemotherapy with FOLFOX4 and surgery versus surgery alone for resectable liver metastases from colorectal cancer (EORTC Intergroup trial 40983): a randomised controlled trial. Lancet. 2008;371(9617): 1007-1016.

3. Weiss L, Grundmann E, Torhorst J, et al. Haematogenous metastatic patterns in colonic carcinoma: an analysis of 1541 necropsies. $J$ Patho. 1986;150(3):195-203.
4. Piedbois P, Buyse M, Kemeny N, et al; Meta-Analysis Group in Cancer. Reappraisal of hepatic arterial infusion in the treatment of nonresectable liver metastases from colorectal cancer. J Natl Cancer Inst. 1996; 88(5):252-258.

5. Ota M, Shimada H, Masui H, et al. Adjuvant hepatic arterial infusion chemotherapy after curative resection for Dukes $\mathrm{C}$ colorectal cancer: a pilot study. Hepatogastroenterology. 2004;51(55):124-127.

6. Andre T, Boni C, Mounedji-Boudiaf L, et al. Oxaliplatin, fluorouracil, and leucovorin as adjuvant treatment for colon cancer. $N$ Engl J Med. 2004;350(23):2343-2351.

7. Kuebler JP, Wieand HS, O'Connell MJ, et al. Oxaliplatin combined with weekly bolus fluorouracil and leucovorin as surgical adjuvant chemotherapy for stage II and III colon cancer: results from NSABP C-07. J Clin Oncol. 2007;25(16):2198-2204.

8. Dzodic R, Gomez-Abuin G, Rougier P, et al. Pharmacokinetic advantage of intra-arterial hepatic oxaliplatin administration: comparative results with cisplatin using a rabbit VX2 tumor model. Anticancer Drugs. 2004; 15(6):647-650.

9. Kern W, Beckert B, Lang N, et al. Phase I and pharmacokinetic study of hepatic arterial infusion with oxaliplatin in combination with folinic acid and 5-fluorouracil in patients with hepatic metastases from colorectal cancer. Ann Oncol. 2001;12(5):599-603.

10. Fiorentini G, Rossi S, Dentico P, et al. Oxaliplatin hepatic arterial infusion chemotherapy for hepatic metastases from colorectal cancer: a phase I-II clinical study. Anticancer Res. 2004;24(3b):2093-2096.

11. Sobin LH, Wittekind C. UICC TNM classification of malignant tumours. 5th ed. New York: Wiley-Liss, Inc.; 1997.

12. Wang MJ, Ping J, Li Y, et al. Prognostic significance and molecular features of colorectal mucinous adenocarcinomas: a strobe-compliant study. Medicine. 2015;94(51):e2350.

13. Siegel R, Ward E, Brawley O, Jemal A. Cancer statistics, 2011: the impact of eliminating socioeconomic and racial disparities on premature cancer deaths. CA Cancer J Clin. 2011;61(4):212-236.

14. Obrand DI, Gordon PH. Incidence and patterns of recurrence following curative resection for colorectal carcinoma. Dis Colon Rectum. 1997;40(1):15-24.

15. Ayoub JP, Hess KR, Abbruzzese MC, Lenzi R, Raber MN, Abbruzzese JL. Unknown primary tumors metastatic to liver. J Clin Oncol. 1998;16(6):2105-2112.

16. Thomas WM, Morris DL, Hardcastle JD. Contact ultrasonography in the detection of liver metastases from colorectal cancer: an in vitro study. Br J Surg. 1987;74(10):955-956. 
17. Taylor I, Machin D, Mullee M, Trotter G, Cooke T, West C. A randomized controlled trial of adjuvant portal vein cytotoxic perfusion in colorectal cancer. Br J Surg. 1985;72(5):359-363.

18. Wolmark N, Rockette H, Wickerham DL, et al. Adjuvant therapy of Dukes' A, B, and C adenocarcinoma of the colon with portal-vein fluorouracil hepatic infusion: preliminary results of National Surgical Adjuvant Breast and Bowel Project Protocol C-02. J Clin Oncol. 1990;8(9): $1466-1475$

19. Ackerman NB, Lien WM, Kondi ES, Silverman NA. The blood supply of experimental liver metastases. I. The distribution of hepatic artery and portal vein blood to "small" and "large" tumors. Surgery. 1969; 66(6):1067-1072.
20. Conway JG, Popp JA, Ji S, Thurman RG. Effect of size on portal circulation of hepatic nodules from carcinogen-treated rats. Cancer Res. 1983;43(7):3374-3378.

21. Archer SG, Gray BN. Vascularization of small liver metastases. $B r$ J Surg. 1989;76(6):545-548.

22. Del Freo A, Fiorentini G, Sanguinetti F, et al. Hepatic arterial chemotherapy with oxaliplatin, folinic acid and 5-fluorouracil in pre-treated patients with liver metastases from colorectal cancer. In Vivo. 2006; 20(6A):743-746.

\section{Publish your work in this journal}

OncoTargets and Therapy is an international, peer-reviewed, open access journal focusing on the pathological basis of all cancers, potential targets for therapy and treatment protocols employed to improve the management of cancer patients. The journal also focuses on the impact of management programs and new therapeutic agents and protocols on

\section{Dovepress}

patient perspectives such as quality of life, adherence and satisfaction. The manuscript management system is completely online and includes a very quick and fair peer-review system, which is all easy to use. Visit http://www.dovepress.com/testimonials.php to read real quotes from published authors.

Submit your manuscript here: http://www.dovepress.com/oncotargets-and-therapy-journal 